\title{
Low threshold L-band mode-locked ultrafast fiber laser assisted by microfiber-based single wall carbon nanotube saturable absorber
}

\begin{abstract}
ABSTRCT
We demonstrate a passively mode-locked erbium-doped fiber laser in L-band wavelength region with low mode-locking threshold employing a $1425 \mathrm{~nm}$ pump wavelength. The mode-locking regime is generated by microfiber-based saturable absorber using carbon nanotube-polymer composite in a ring cavity. This carbon nanotube saturable absorber shows saturation intensity of $9 \mathrm{MW} / \mathrm{cm}^{2}$. In this work, mode-locking laser threshold is observed at $36.4 \mathrm{~mW}$ pump power. At the maximum pump power of $107.6 \mathrm{~mW}$, we obtain pulse duration at full-width half-maximum point of $490 \mathrm{fs}$ and time bandwidth product of 0.33 , which corresponds to 3-dB spectral bandwidth of $5.8 \mathrm{~nm}$. The pulse repetition rate remains constant throughout the experiment at 5.8 $\mathrm{MHz}$ due to fixed cavity length of $35.5 \mathrm{~m}$. Average output power and pulse energy of $10.8 \mathrm{~mW}$ and $1.92 \mathrm{~nJ}$ are attained respectively through a $30 \%$ laser output extracted from the mode-locked cavity. This work highlights the feasibility of attaining a low threshold mode-locked laser source to be employed as seed laser in L-band wavelength region.
\end{abstract}

Keyword: Ultrashort pulse; Pulsed fiber laser; Carbon nanotube. 